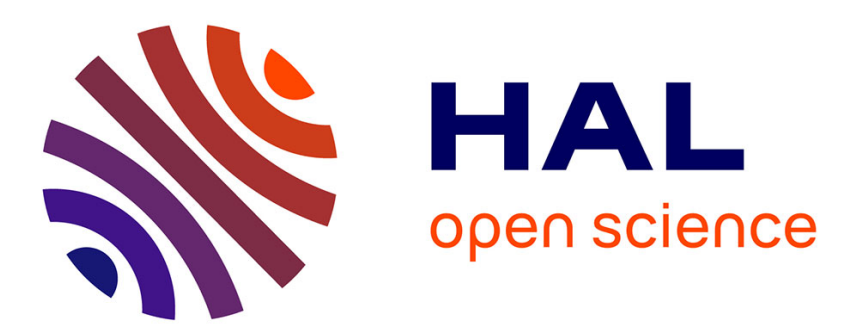

\title{
Algebraic switching time identification for a class of linear hybrid systems
}

\author{
Yang Tian, Thierry Floquet, Lotfi Belkoura, Wilfrid Perruquetti
}

\section{To cite this version:}

Yang Tian, Thierry Floquet, Lotfi Belkoura, Wilfrid Perruquetti. Algebraic switching time identification for a class of linear hybrid systems. ADHS'09 - IFAC Conference on Analysis and Design of Hybrid Systems, Sep 2009, Saragosse, Spain. 10.1016/j.nahs.2010.07.003 inria-00424440

\section{HAL Id: inria-00424440 \\ https://hal.inria.fr/inria-00424440}

Submitted on 15 Oct 2009

HAL is a multi-disciplinary open access archive for the deposit and dissemination of scientific research documents, whether they are published or not. The documents may come from teaching and research institutions in France or abroad, or from public or private research centers.
L'archive ouverte pluridisciplinaire HAL, est destinée au dépôt et à la diffusion de documents scientifiques de niveau recherche, publiés ou non, émanant des établissements d'enseignement et de recherche français ou étrangers, des laboratoires publics ou privés. 


\title{
Algebraic switching time identification for a class of linear hybrid systems
}

\author{
Yang TIAN* ${ }^{*}$ Thierry FLOQUET ${ }^{*}$ Lotfi BELKOURA ${ }^{* *}$ \\ Wilfrid PERRUQUETTI* \\ * LAGIS (CNRS, UMR 8146) \& ALIEN Project (INRIA Lille-Nord Europe), \\ École Centrale de Lille, BP 48, Cité Scientifique, 59650 Villeneuve d'Ascq, \\ France(e-mail: yang.tian@inria.fr, thierry.floquet@ec-lille.fr, \\ wilfrid.perruquetti@ec-lille.fr) \\ ** LAGIS (CNRS, UMR 8146) \& ALIEN Project (INRIA Lille-Nord Europe), \\ Université des Sciences et Technologies de Lille, 59650 Villeneuve d'Ascq, \\ France (e-mail: lotfi.belkoura@univ-lille1.fr)
}

\begin{abstract}
In this paper, a method for the finite time estimation of the switching times in linear switched systems is proposed. The approach is based on algebraic tools (differential algebra, module theory and operational calculus) and distribution theory. Switching time estimates are given by explicit algebraic formulae that can be implemented in a straightforward manner using standard tools from computational mathematics. Simulations illustrate the proposed techniques.
\end{abstract}

Keywords: Linear systems, switched systems, switching time estimation, distribution theory.

\section{INTRODUCTION}

Many systems encountered in practice exhibit switchings between several subsystems, inherently by nature, such as when a physical plant has the capability of undergoing several operational modes, or as a result of the controller design, such as in switching supervisory control.

Switched systems can be seen as higher-level abstractions of hybrid systems, obtained by neglecting the details of the discrete behavior. A switched system is composed of a family of dynamical (linear or nonlinear) subsystems and a rule, called the switching law, that orchestrates the switching between them (see Liberzon (2003); Sun and Ge (2005) for surveys). In the recent years, there has been an increasing interest in the control problems of switched systems due to their significance from both a theoretical and practical point of view. Important results for switched systems have been achieved for problems including stability Agrachev and Liberzon (2001); Boscain (2006); Branicky (1998); Mancilla-Aguilar and García (2000); Vu and Liberzon (May, 2005), stabilization Moulay et al. (2007); Persis et al. (2004); Wicks et al. (1998); Zhai et al. (2003), tracking Bourdais et al. (2007) or controllability Sun et al. (May, 2002); Xie et al. (2002).

Observability and state estimation is a key problem for such systems because both the active mode and the continuous state have to be estimated and this during a finite time interval. The notion of state estimation for switched systems was introduced in Ackerson and Fu (1970). Observability notions for some classes of hybrid systems such as switched linear systems has been discussed and characterized in recent works such as Babaali and Pappas (2005), Santis et al. (2003), Vidal et al. (2003). The problem is to recover from available measurements the state of the system and/or the switching signal, and eventually the switching time. Different observation and identification methods have been performed during the last years (Alessan- dri and Coletta (2001); Balluchi et al. (2002); Domlan et al. (2007); Fantuzzi et al. (2002); Hashambhoy and Vidal (2005); Heemels et al. (2001); Huang et al. (2004); Paoletti et al. (2007); Saadaoui et al. (2006)). Usually, the hybrid observer consists of two parts: an index estimator of the current active sub-model and a continuous observer that estimates, asymptotically in most cases, the continuous state of the hybrid system.

The aim of this paper is to estimate in "real-time" the switching time sequence of some class of switched linear systems with the knowledge of the continuous state only. The possibility to have finite time estimate for that kind of systems is clearly important, not to say crucial. The approach considered here takes root in recent works developed by Fliess and Sira-Ramírez (2003) for parameter identification of linear time-invariant systems. This method is based on algebraic tools (differential algebra, module theory and operational calculus) and results in finite estimates given by explicit algebraic formulae that can be implemented in a straightforward manner using standard tools from computational mathematics. Those results have been extended to the problems of closed-loop parametric estimation in Fliess and Sira-Ramírez (2008), state estimation of linear systems with time-varying parameters in Tian et al. (2008) or with delays in Belkoura et al. (2006), fault diagnosis in Fliess et al. (2004), nonlinear systems with unknown inputs in Barbot et al. (2007) or some class of linear infinite dimensional systems governed by partial differential equations in Rudolph and Woittennek (2008). This approach was also applied in Fliess et al. (2008) for the estimation of the index corresponding to the current active subsystem, and the state variable of this subsystem. In this paper, the algebraic approach is extended to the problem of the finite time identification of the switching occurrences. Using some properties of the distribution theory, the switching time estimation is given by an explicit formulae, as a function of the integral of the output, in order to attenuate the influence of measurement noises. 


\section{PROBLEM STATEMENT}

In this work, we study a class of linear hybrid systems called switched linear systems (definition see Vidal et al. (2003)), i.e. systems whose evolution is determined by a collection of linear models ( $Q$ subsystems) with continuous state $x \in$ $\mathbb{R}^{n}$ connected by switches among a number of discrete states $q \in I_{Q} \triangleq\{1, \ldots, Q\}$, modeled by linear ordinary differential equations of the following form:

$$
\dot{x}=A_{q} x,
$$

$A_{q} \in \mathbb{R}^{n \times n}$ are constant matrices. For the sake of convenience and without loss of generality, it is assumed that at each time $t \in \mathbb{R}$ only one discrete event can act on the system. It is also assumed that there is no jump, i.e. that if $t_{k}$ is a switching instant

$$
\lim _{t \rightarrow t_{k}^{-}} x(t)=\lim _{t \rightarrow t_{k}^{+}} x(t)
$$

The objective of this paper is to estimate on line the switching times of the system (1) using the dynamics of the continuous state only. It will be assumed that all the state is available for measurement. The more general case with partial state measurements will be studied in further work. The method that will be developed will involve high order time derivatives of $x$. Because of the presence of non smooth dynamics, derivation has to be understood in the distribution sense.

\subsection{Distribution Framework}

We recall here some standard definitions and results from distribution theory developed in Schwartz (1966), and fix the notations to be used in the sequel. The space of $C^{\infty}$-functions having compact support in an open subset $\Omega$ of $\mathbb{R}$ is denoted by $\mathscr{D}(\Omega)$, and $\mathscr{D}^{\prime}(\Omega)$ is the space of distributions on $\Omega$, i.e., the space of continuous linear functionals on $\mathscr{D}(\Omega)$.

When concentrated at a point $\{\tau\}$, the Dirac distribution $\delta(t-$ $\tau)$ is written $\delta_{\tau}$.

Functions are considered through the distributions they define and are therefore indefinitely differentiable. Hence, if $y$ is a continuous function except at a point $a$ with a finite jump $\sigma_{a}$, its derivative writes

$$
\dot{y}=d y / d t+\sigma_{a} \delta_{a},
$$

where $d y / d t$ is the distribution stemming from the usual derivative of $y$.

A distribution is said to be of order $r$ if it acts continuously on $C^{r}$-functions but not on $C^{r-1}$-functions. Measures and functions are of order 0. By virtue of Schwartz Theorem (see Schwartz (1966)), $\alpha \times T=0$ for any smooth function $\alpha$ whose derivatives of appropriate order vanish on the support of a given distribution $T \in \mathscr{D}^{\prime}(\Omega)$. In particular, one has for the Dirac distribution ${ }^{1}$ :

$$
\begin{aligned}
& f(t) \cdot \delta_{t_{i}}=0, \forall f \in C^{\infty}(\mathbb{R}) \text { if } f\left(t_{i}\right)=0, \\
& f(t) \cdot \delta_{t_{i}}=f\left(t_{i}\right) \cdot \delta_{t_{i}}, \forall f \in C^{\infty}(\mathbb{R}) \text { if } f\left(t_{i}\right) \neq 0 .
\end{aligned}
$$

\footnotetext{
1 The existence of the product $f(t) \cdot \delta_{t_{i}}$ is ensured as soon as $f$ is a $C^{\infty}$-function.
}

\section{SWITCHING TIME IDENTIFICATION}

\subsection{The case of two subsystems and one commutation}

In this case, $q \in\{1,2\}$. Assume that the system (1) switches from the subsystem 1 to the subsystem 2 at the time $t_{c}$. Then, the dynamical behavior of the system can be rewritten as follows:

$$
\begin{aligned}
& \dot{x}=\Gamma(t) x \\
& \Gamma(t)=A_{1} H(t)+A_{2}(1-H(t))
\end{aligned}
$$

with the function $H$ given by:

$$
H(t)= \begin{cases}1, & 0 \leq t<t_{c} \\ 0, & t_{c} \leq t<T .\end{cases}
$$

The aim is to get a finite time estimation of the switching time $t_{c}$ when the system commutes from $q=1$ to $q=2$.

Assume that $A_{1}$ and $A_{2}$ are two square commuting matrices and consider the following change of variable:

$$
z=e^{G t} x
$$

with $G=-\frac{A_{1}+A_{2}}{2}$. One has:

$$
\dot{z}=G e^{G t} x+e^{G t} \dot{x}=G z+e^{G t} \Gamma(t) x=\left(G+e^{G t} \Gamma(t) e^{-G t}\right) z
$$

This leads to

$$
\dot{z}=M(t) z
$$

with

$$
M(t)=\left(G+e^{G t} A_{1} e^{-G t}\right) H(t)+\left(G+e^{G t} A_{2} e^{-G t}\right)(1-H(t)) .
$$

Since $A_{1}$ and $A_{2}$ are two square commuting matrices, one gets:

$$
\begin{aligned}
M(t) & =\left(G+A_{1}\right) H(t)+\left(G+A_{2}\right)(1-H(t)) \\
& =\frac{A_{1}-A_{2}}{2} H(t)+\frac{A_{2}-A_{1}}{2}(1-H(t)) \\
& =-A H(t)+A(1-H(t))
\end{aligned}
$$

with $A=\frac{A_{2}-A_{1}}{2}$. The choice of the change of coordinates (4) is motivated by the fact that the subsequent computations involve $M^{2}=A^{2}$ which is a known quantity independent of the switching time.

Differentiating (5) over the interval $t \in[0, T$, one has:

$$
\begin{aligned}
& \ddot{z}-A^{2} z=-2 A \delta_{0} z(0), \quad 0 \leq t<t_{c} \\
& \ddot{z}-A^{2} z=-2 A \delta_{0} z(0)+2 A \delta_{t_{c}} z\left(t_{c}\right), \quad t_{c} \leq t<T
\end{aligned}
$$

which can be rewritten in the following compact form:

$$
\ddot{z}-A^{2} z=-2 A \delta_{0} z(0)+2 A(1-H(t)) \delta_{t_{c}} z\left(t_{c}\right), \quad 0 \leq t<T
$$

Note that if the initial subsystem is $q=2$, a similar expression can be obtained as follows:

$$
\ddot{z}-A^{2} z=2 A \delta_{0} z(0)-2 A(1-H(t)) \delta_{t_{c}} z\left(t_{c}\right), 0 \leq t<T
$$

the terms in the right hand have the opposite signum. As it will be seen hereafter, this slight difference has no influence on the result because the right hand side of (6) will be canceled by suitable algebraic manipulations. Thus the proposed method does not need the knowledge of the current mode.

\subsection{Explicit computation of the switching instant}

Take any function with the following properties:

(i) $f\left(t, t_{c}\right) \delta_{t_{c}}=0$,

(ii) $f\left(0, t_{c}\right)=\dot{f}\left(0, t_{c}\right)=0$. 
Multiplying (6) by $f\left(t, t_{c}\right)$, one obtains:

$$
f\left(t, t_{c}\right)\left(\ddot{z}-A^{2} z\right)=0 .
$$

Integrating (7) from 0 to $t_{c}<t \leq T$ leads to

$$
\int_{0}^{t} f\left(\tau, t_{c}\right)\left(\ddot{z}(\tau)-A^{2} z(\tau)\right) d \tau=0
$$

Integration by parts gives:

$$
\begin{aligned}
\int_{0}^{t} f\left(\tau, t_{c}\right) \ddot{z}(\tau) d \tau & =\left[f\left(\tau, t_{c}\right) \dot{z}(\tau)\right]_{\tau=0}^{\tau=t}-\int_{0}^{t} \dot{f}\left(\tau, t_{c}\right) \dot{z}(\tau) d \tau \\
& =f\left(t, t_{c}\right) \dot{z}(t)-\int_{0}^{t} \dot{f}\left(\tau, t_{c}\right) \dot{z}(\tau) d \tau
\end{aligned}
$$

and

$$
\begin{aligned}
\int_{0}^{t} \dot{f}\left(\tau, t_{c}\right) \dot{z}(\tau) d \tau & =\left[\dot{f}\left(\tau, t_{c}\right) z(\tau)\right]_{\tau=0}^{\tau=t}-\int_{0}^{t} \ddot{f}\left(\tau, t_{c}\right) z(\tau) d \tau \\
& =\dot{f}\left(t, t_{c}\right) z(t)-\int_{0}^{t} \ddot{f}\left(\tau, t_{c}\right) z(\tau) d \tau
\end{aligned}
$$

Hence

$$
\begin{aligned}
& \int_{0}^{t} f\left(\tau, t_{c}\right) \ddot{z}(\tau) d \tau \\
& \quad=f\left(t, t_{c}\right) \dot{z}(t)-\dot{f}\left(t, t_{c}\right) z(t)+\int_{0}^{t} \ddot{f}\left(\tau, t_{c}\right) z(\tau) d \tau
\end{aligned}
$$

Integrating one more time (8) from 0 to $t_{c}<t \leq T$ and using (9) leads to the following relation:

$$
\begin{aligned}
\int_{0}^{t}\left[f\left(\tau, t_{c}\right) \dot{z}(\tau)-\dot{f}\left(\tau, t_{c}\right) z(\tau)\right] d \tau \\
+\int_{0}^{t} \int_{0}^{\tau_{1}}\left(\ddot{f}\left(\tau, t_{c}\right)-A^{2} f\left(\tau, t_{c}\right)\right) z(\tau) d \tau d \tau_{1}=0
\end{aligned}
$$

Thus, using the property

$$
\int_{0}^{t} \int_{0}^{t_{v-1}} \cdots \int_{0}^{t_{1}} x(\tau) d t_{v-1} \cdots d t_{1} d \tau=\int_{0}^{t} \frac{(t-\tau)^{v-1}}{(v-1) !} x(\tau) d \tau
$$

one has:

$$
\begin{aligned}
f\left(t, t_{c}\right) z & (t)-2 \int_{0}^{t} \dot{f}\left(\tau, t_{c}\right) z(\tau) d \tau \\
& +\int_{0}^{t}(t-\tau)\left(\ddot{f}\left(\tau, t_{c}\right)-A^{2} f\left(\tau, t_{c}\right)\right) z(\tau) d \tau=0 .
\end{aligned}
$$

The following function $f\left(t, t_{c}\right)=t^{2}\left(t-t_{c}\right)$ satisfies the two properties (i)-(ii) and

$$
\begin{aligned}
& \dot{f}\left(t, t_{c}\right)=t\left(3 t-2 t_{c}\right) \\
& \ddot{f}\left(t, t_{c}\right)=6 t-2 t_{c}
\end{aligned}
$$

Using the result of (10), the estimate of $t_{c}$ is given by the following formula:

$$
D(t, z) t_{c}=N(t, z)
$$

with

$$
\begin{aligned}
& N(t, z, A)=t^{3} z(t)-\int_{0}^{t} 6 \tau^{2} z(\tau) d \tau+\int_{0}^{t}(t-\tau)\left(6 \tau I-A^{2} \tau^{3}\right) z(\tau) d \tau \\
& D(t, z, A)=t^{2} z(t)-\int_{0}^{t} 4 \tau z(\tau) d \tau+\int_{0}^{t}(t-\tau)\left(2 I-A^{2} \tau^{2}\right) z(\tau) d \tau
\end{aligned}
$$

where $I \in \mathbb{R}^{n \times n}$ is the unit matrix. Note that $N(t, z, A)$ and $D(t, z, A)$ are column vectors of dimension $n$. So

$$
t_{c}=\frac{N_{g}(t, z, A)}{D_{g}(t, z, A)},
$$

for any $1 \leq g \leq n$, where $N_{g}(t, z, A)$ and $D_{g}(t, z, A)$ are the components of $\bar{N}(t, z, A)$ and $D(t, z, A)$, respectively.
Thus, one obtains the exact formula for the identification of the switching time $t_{c}$. Hereafter, the method is extended to the problem of the identification of the switching times between an arbitrary number of subsystems.

\subsection{The case of $Q$ subsystems and $S$ commutations}

Assume that every pair of matrices $A_{i}$ and $A_{j}\left(i, j \in I_{Q}\right.$ and $\left.i \neq j\right)$ in system (1) are commuting. It has been seen that an estimator $E_{i, j}$ that computes the quantities $D\left(t, z, A_{i, j}\right)$ and $N\left(t, z, A_{i, j}\right)$, with $A_{i, j}=\frac{A_{j}-A_{i}}{2}$ can be used to determine a switching time $t_{i, j}$ that occurs between the two subsystems $i$ and $j$ (either from mode $i$ to mode $j$ or from mode $j$ to mode $i$ ).

Hence, in order to identify all the switches among $Q$ modes, one can use $C_{Q}^{2}$ estimators in parallel. Then, the output signals of each estimator can be analyzed as follows to determine the occurrence of a switch and its associated mode. Indeed, assume that the system (1) is in the mode $i$ for $t \in\left[t_{0}, t_{i, j}\right.$ [ and in mode $j$ for $t \in\left[t_{i, j}, T\right.$ [, where times $t_{0}$ and $T$ stands for other switch occurrences. Then, one has:

(1) $D_{g}\left(t, z, A_{i, j}\right)=0$ and $N_{g}\left(t, z, A_{i, j}\right)=0$ for $t \in\left[t_{0}, t_{i, j}[\right.$ : in practice several successive points have to be tested before the switching instant;

(2) $D_{g}\left(t, z, A_{i, j}\right)$ and $N_{g}\left(t, z, A_{i, j}\right)$ are straight lines for $t \in$ $\left[t_{i, j}, T[:\right.$ in practice several points have to be used to detect the slope of the line is constant.

(3) for $t \in\left[t_{i, j}, T\right.$ [ the ratio of $\frac{N_{g}\left(t, z, A_{i, j}\right)}{D_{g}\left(t, z, A_{i, j}\right)}$ is constant and equal to $t_{i, j}$ : to detect when this ratio is constant, successive values within a boundary of a given thickness are needed.

Properties (1) and (2) can be seen from the fact that Dirac distributions in equation (6) are integrated twice. Thus, the estimator that fulfills those three conditions provides the switching instant as well as the index of the subsystems between which the switch occurs. Furthermore, with the knowledge of the first active mode, the sequence of the all active models can be estimated.

The implementation of the numerical algorithm which allows to identify all the switching instants is done on a sliding window as follows:

\section{function detection of the switching time $t_{c}$}

read $x\left(k T_{e}\right)$

$T_{e}$ : sample time;

$h$ : number of points chosen to verify the constraints with $h \cdot T_{e}$ less than the dwell time of the system;

$t_{s}=0:$ left bound of the integration window;

$m=$ length $(x)$ : length of the sampled state $x$;

$z_{i, j}\left(k T_{e}\right)=e^{-\frac{A_{i}+A_{j}}{2}} x\left(k T_{e}\right):$ new state

$$
\text { for } k=h: m
$$

Reset all the components of $N\left(t, z, A_{i, j}\right)(k)$ and $D\left(t, z, A_{i, j}\right)(k)$ to zero

$$
\text { for } i=t_{s}: k
$$

Approximate numerically the integrals as sums using the trapezoidal rule 
end

Compute the value of $N\left(t, z, A_{i, j}\right)(k)$ and $D\left(t, z, A_{i, j}\right)(k)$ in an integration window with (11)

if an estimator satisfies the three detection criterions update the integration window: $\mathbf{t}_{\mathbf{s}} \longleftarrow \mathbf{k}-\mathbf{h}$;

print this estimator and the active model.

else

break;

end

print the switching time

end

The numerical implementation of the above obtained algorithm has also to address the following points:

- integration window: when none of the $C_{Q}^{2}$ estimator detect the commutation, algorithm will calculate the value of $N\left(t, z, A_{i, j}\right)(k), D\left(t, z, A_{i, j}\right)(k)$ in a new integration window $(k \longleftarrow k+1)$ whose width is one sample time larger than the old one (lower bound of the integration no change while upper bound increase one sampling), until a switching instant is detected with one of the estimator.

- update: when $t_{c}$ is detected, one has to update the scheme by resetting the integral computation and replacing the lower bound of the integration with $t_{c}$ in order to identify the next switching time (for this in the integral approximation).

\section{EXAMPLE}

Consider a linear switched system consisting of three linear time invariant scalar systems given by:

$$
\begin{aligned}
& A_{1}=-8 \\
& A_{2}=-1 \\
& A_{3}=0.4
\end{aligned}
$$

The switching instants of this switched system are detected using the algorithm proposed in Section 3 with $T_{e}=0.001 \mathrm{~s}$ and 3 estimators $\left(C_{3}^{2}\right)$. The first active model is subsystem 1 and the switching sequences among the subsystems are $A_{1} \longrightarrow A_{2} \longrightarrow$ $A_{3} \longrightarrow A_{1} \longrightarrow A_{2} \longrightarrow A_{3} \longrightarrow A_{2} \longrightarrow A_{1}$. Since estimator $E_{1,2}$ detects the commutation between $A_{1} \longleftrightarrow A_{2}$, estimator $E_{2,3}$ corresponds to the commutation between $A_{2} \longleftrightarrow A_{3}$ and estimator $E_{1,3}$ detects the switch between $A_{1} \longleftrightarrow A_{3}$, it is known that estimators $E_{1,2} \longrightarrow E_{2,3} \longrightarrow E_{1,3} \longrightarrow E_{1,2} \longrightarrow$ $E_{2,3} \longrightarrow E_{2,3} \longrightarrow E_{1,2}$ work respectively. The switching times are assumed to occur at: $t_{1}=0.2 s, t_{2}=1.2 s, t_{3}=2.4 s, t_{4}=2.6 s$, $t_{5}=3.6 s, t_{6}=4 s$ and $t_{7}=4.9 s$. In the simulation, we choose 30 samplings to identify the switching time, so the dwell time has to be at least larger than $0.03 \mathrm{~s}$.

Fig. 1 shows that the estimator performs well. Note that $t_{4}$ occurs shortly $(0.2 s)$ after $t_{3}$ but that the algorithm can detect it accurately.
Fig. 2 gives the estimation of the sequence of active model. It can be seen that the estimated switched system $S_{e}$ tracks exactly the real switched system $S_{r}$.

Note that identification of the switching time does not need the knowledge of the first active mode, but is just required in order to obtain the sequence of the active model.

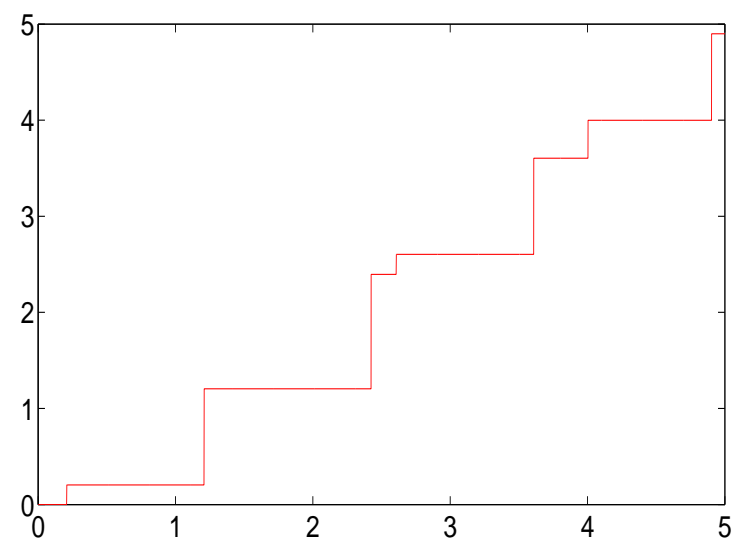

Fig. 1. Identification of the switching times $t_{c}$.

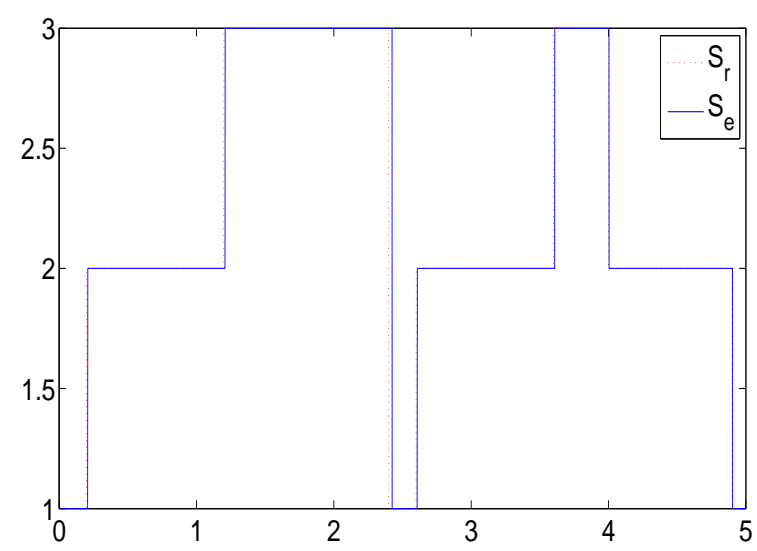

Fig. 2. Estimation of the active model.

\section{CONCLUSION}

In this paper, an algebraic approach for switching time estimation of a class of hybrid systems has been introduced. An explicit algorithm which computes on-line the switching time instants in a fast way has been derived. In future works, this approach will be extended to more general cases with partial state measurements.

\section{REFERENCES}

Ackerson, G.A. and Fu, K.S. (1970). On state estimation in switching environnements. IEEE Trans. Automat. Control, 15(1), 10-17.

Agrachev, A.A. and Liberzon, D. (2001). Lie-algebraic stability criteria for switched systems. SIAM J. Control Optimiz., 40.

Alessandri, A. and Coletta, P. (2001). Design of Luenberger Observers for a Class of Hybrid Linear Systems. In hybrid systems: Computation and Control, Springer Verelag, 2034, $7-18$. 
Babaali, M. and Pappas, G.J. (2005). Observability of switched linear systems in continuous time. Lecture Notes in Computer Science (Hybrid Systems: Computation and Control), 3414, 103-117.

Balluchi, A., Benvenuti, L., Benedetto, M.D.D., and Sangiovanni-Vincentelli, A.L. (2002). Design of observers for hybrid systems. Lecture notes in computer Science 2289, Springer Verelag, 77-89.

Barbot, J.P., Fliess, M., and Floquet, T. (2007). An algebraic framework for the design of nonlinear observers with unknown inputs. In 46th IEEE Conference on Decision and Control. New Orleans, Louisiana, USA.

Belkoura, L., Richard, J.P., and Fliess, M. (2006). On-line identification of systems with delayed inputs. In 17th Symposium on Mathematical Theory of Networks and Systems (MTNS). Kyoto, Japon.

Boscain, U. (2006). Stability of planar switched systems: The linear single input case. SIAM J. Control Optimiz., 73, 89112.

Bourdais, R., Fliess, M., Join, C., and Perruquetti, W. (2007). Towards a model-free output tracking of switched nonlinear systems. 7th IFAC Symposium on Nonlinear Control Systems, NOLCOS 2007, 637-642. Pretoria, South Africa.

Branicky, M.S. (1998). Multiple Lyapunov functions and other analysis tools for switched and hybrid systems. IEEE Trans. Automat. Control., 43, 475-482.

Domlan, E.A., Ragot, J., and Maquin, D. (2007). Switching systems: Active mode recognition, identification of the switching law. Journal of Control Science and Engineering, 11.

Fantuzzi, C., Simani, S., Beghelli, S., and Rovatti, R. (2002). Identification of piecewise affine models in noisy environment. Int. J. of Control, 75(18), 1472-1485.

Fliess, M., Join, C., and Perruquetti, W. (2008). Real-time estimation for switched linear systems. 47th IEEE Conference on Decision and Control.

Fliess, M., Join, C., and Sira-Ramírez, H. (2004). Robust residual generationfor linear fault diagnosis: an algebraic setting with examples. Int. J. of Control, 77, 1223-1242.

Fliess, M. and Sira-Ramírez, H. (2003). An algebraic framework for linear identification. ESAIM Control Optim. Calc. Variat., 9, 151-168.

Fliess, M. and Sira-Ramírez, H. (2008). Closed-loop parametric identification for continuous-time linear systems via new algebraic techniques.

Hashambhoy, Y. and Vidal, R. (2005). Recursive identification of switched arx models with unknown number of models and unknown orders. Proceedings of the Joint 44th IEEE Conference on Decision and Control and European Control Conference. Sevilla, Spain.

Heemels, W., Schutter, B.D., and Bemporad, A. (2001). Equivalence of hybrid dynamical models. Automatica, 37(7), 1085-1091.

Huang, K., Wagner, A., and Ma, Y. (2004). Identification of hybrid linear time-invariant systems via subspace embedding and segmentation (ses). $43^{\text {th }}$ IEEE Conf. Decision Control. Atlantis, Paradise Island, Bahamas.

Liberzon, D. (2003). Switching in systems and control. Systems \& Control: Foundation \& Applications. Birkhauser.

Mancilla-Aguilar, J.L. and García, R.A. (2000). A converse Lyapunov theorem for nonlinear switched systems. Systems Control Lett., 41, 67-71.
Moulay, E., Bourdais, R., and Perruquetti, W. (2007). Stabilization of nonlinear switched systems using control Lyapunov functions. Nonlinear Analysis: Hybrid Systems, 1, 482-490.

Paoletti, S., Juloski, A.L., Ferrari-Trecate, G., and Vidal, R. (2007). Identification of hybrid systems: a tutorial. European Journal of Control, 13(2-3).

Persis, C.D., Santis, R.D., and Morse, A.S. (2004). Supervisory control with state-dependent dwell-time logic and constraints. Automatica, 40(2), 269-275.

Rudolph, J. and Woittennek, F. (2008). An algebraic approach to parameter identification in linear infinite dimensional systems. In IEEE Mediterranean Conference on Control and Automation. Ajaccio, France.

Saadaoui, H., Manamanni, N., Djemaï, M., Barbot, J.P., and Floquet, T. (2006). Exact differentiation and sliding mode observers for switched lagrangian systems. Nonlinear Analysis Theory, Methods and Applications, 65(5), 1050-1069.

Santis, E.D., Benedetto, M.D.D., and Pola, G. (2003). On observability and detectability of continuous-time linear switching systems. Proc. 42nd IEEE Conf. on Decision and Control, 5777-5782. Maui, Hawaii, USA.

Schwartz, L. (1966). Théorie des distributions. 2nd ed. Hermann.

Sun, Z., Ge, S., and Lee, T. (May, 2002). Controllability and reachability criteria for switched linear control. Automatica, 38(5), 775-786.

Sun, Z. and Ge, S.S. (2005). Switched linear systems: Control and design. London: Springer-Verlag.

Tian, Y., Floquet, T., and Perruquetti, W. (2008). Fast state estimation in linear time-variant systems: an algebraic approach. In 47th IEEE Conference on Decision and Control. Cancun Mexique.

Vidal, R., Chiuso, A., Soatto, S., and Sastry, S. (2003). Observability of linear hybrid systems. Lecture Notes in Computer Science (Hybrid Systems Computation and Control), 2623, 526-539.

Vu, L. and Liberzon, D. (May, 2005). Common Lyapunov functions for families of commuting nonlinear systems. Systems Control Lett., 54(5), 405-416.

Wicks, M., Peleties, P., and DeCarlo, R. (1998). Switched controller synthesis for the quadratic stabilization of a pair of unstable linear systems. European Journal of Control, 4(2), $140-147$.

Xie, G., Zheng, D., and Wang, L. (2002). Controllability of switched linear systems. IEEE Trans. Automat. Control, 47(8), 1401-1405.

Zhai, G., Lin, H., and Antsaklis, P.J. (2003). Quadratic stabilizability of switched linear systems with polytopic uncertainties. Internat. J. Control, 76(7), 747-753. 